\title{
Innovation Opportunity for 5G Technologies: A Survey
}

\author{
Suniti Purbey \\ Assistant Professor \\ Amity Institute of Information Technology \\ Amity University Chhattisgarh, Raipur \\ spurbey@rpr.amity.edu
}

\section{Abstract}

The "not withal formally defined" $5 \mathrm{G}$ commonplace leads to the consequence that its relevant technological innovation continues to be wide open. For distinctive innovations probability, we have a tendency to should discover the actual technical area to contribute to the technological development of 5G. This paper discusses a framework responsive the foremost question: inside that technological area one may contribute to the innovation? The answer shall profit countries, firms, universities and analysis institute that intends to contribute to the formulation of official $5 \mathrm{G}$ commonplace. First, we've got a bent to review the key technologies of fifth generation mobile communication technology (5G). Present and skill of the network unit main technical focus. A flat IP-based network thought was reviewed, additionally as psychological feature radio technology to reach the terminal that have AI. BDMA technology was planned to help achieving system efficiency in terms of multiple access system. Second, we've got a bent to familiar technological challenges, that specialise in issues $\}$ related to security and issues to have an effect on restricted frequency spectrum resources. Afterward, we've got a bent to map the innovation probability supported technical area that's recently unconcealed in analysis article. We've got a bent to terminated that innovation opportunities lies on the analysis about security, network, technological implementation and applications issues.

Index Terms - 5G; innovation; normal

\section{INTRODUCTION}

Today, changes inside the realm of telecommunication technology have occurred and might keep happening. Mobile telecommunication grows from initial generation, spoken as $1 \mathrm{G}$, to $2 \mathrm{G}, 3 \mathrm{G}$, and presently to the fourth generation that additionally in implementation stage in several countries, 4G. Every generation of technology have several variations and innovations.

5G may well be a language thatl's used for the fifth generation mobile technology. Telecommunication companies or standardization bodies of telecommunication like 3GPP, WiMax Forum, or ITU-R haven't issued the official customary for 5G [1]. The absence of official customary makes the $5 \mathrm{G}$ have limitless prospects. However several expectations are raised concerning but $5 \mathrm{G}$ need to and might be.

The 5G technology is expected to complete the 4G technology and provide solutions to the shortage arising from 4G technology. This technology are planning to be a greenhorn technology that produces users able to access altogether completely different Radio Access 
Technologies (RATs) victimization one mobile [2]. 5G has been projected to assemble the current wireless Associate in Nursing wired communication techniques into Associate in Nursing all science (Internet Protocol) high performance worldwide network [3]. 5G technology will facilitate perfecting World Wide Wireless web (WWWW) [4]. WWWW itself is an effort to make a circumstance where subscriber can saver the good quality and quick access of web, dynamic movement, favourable Bit Error relation (BER) and nice security as on wired communications in their wireless communication devices. Limitations of frequency resources making $5 \mathrm{G}$ shall have a technical development that uses completely different resources than frequency/time resources therefore on extend a capability of the system [1].

In this paper, we have a tendency to tend to do and confirm innovation probability of the $5 \mathrm{G}$ technological development. It explored the fundamental literature framework to answer a question: throughout that technological area one may contribute to the innovation? The answer shall profit countries, firms, universities and analysis institute that intends to contribute to the formulation of official $5 \mathrm{G}$ customary.

The second section of this paper provides the review of the key technologies of fifth generation mobile communication technology (5G). The third section presents our identification of technological challenges, specializing in issues $\}$ related to security and issues to injure restricted frequency spectrum resources. Inside the fourth section, we have a tendency to tend to mapped the innovation probability supported technological area that's recently unconcealed in analysis article.

\section{REVIEW OF 5G KEY TECHNOLOGIES}

There unit several key technologies that unit expected to help fulfilling the requirement of improvement for 5G. Those unit Flat science based totally Network and psychological feature Radio (CR).

\section{A Flat science based totally Network}

Previous works by Toni Janevski from University Sv Kiril I Metodij define the essential thought of $5 \mathrm{G}$ mobile network that's seen as user-centric thought instead of operator-centric as in $3 \mathrm{G}$ or service-centric thought as seen for $4 \mathrm{G}$. The $5 \mathrm{G}$ itinerant is supposed as Associate in Nursing open platform on altogether completely different layers, from physical layer up to the applying [5].

The network layer at $5 \mathrm{G}$ networks are planning to be divided into several sub-layers to provide all-IP property anywhere and anytime. The employment of the online Protocol (IP) inside the network layer is inevitable, given the data science system is that the simplest and most used system to support and expand the network layer recently. All science Network (AIPN) system has started well since the event of LTE.

All science Network (AIPN) system has started well since the event of LTE as Associate in Nursing Associate in nursing evolution of the 3GPP system. Flat science Network is also a key thought that l's expected to make $5 \mathrm{G}$ acceptable to all or any or any forms of technology [1]. Flat science style provides the thanks to spot each device victimization symbolic names, in distinction to the hierarchal style ordinarily utilized within the usual science address [1]. With the shift to flat science architectures, mobile operators are planning to be able to [1]:

- Shrink the number of network components inside the data path, thereby reducing operations costs and price.

- Rough the worth of service delivery from the quantity of data thatl's sent to equate infrastructure capabilities to the needs of rising applications

- Minimize system latency and modify applications with a lower tolerance for delay; forthcoming latency enhancements on the link can also be altogether accomplished. 
- Evolve radio access and packet core networks severally of each completely different to understand larger development and build higher flexibility in network springing up with and preparation.

- Develop a flexible core network which will be the thought of innovative services for mobile and generic science access network.

Produce a platform which can modify mobile broadband operators to be competitive with wired networks in terms valuable and performance.

IPv6 is that absolutely the best system which will support a flat science network for technology implementation 5G later. Info sciencev6 is that the newest revision of the information science system is expected to beat the shortcomings of the forerunner version of IPv4. Each device will then have a tough and quick IPv6 address, and multiple addresses Care of Address (CoA). The number of CoA for the device is per the number of access networks where device is connected. There unit 3 sub-layers of the network layer, the lower network layer, middleware layer and thus the upper network layer. Lower network layer use CoA, middleware network layer interprets CoA into IPv6 thus the upper network layer victimization IPv6 addresses.

Device is expected to be provided by a spread of selections therefore on urge the foremost effective wireless affiliation in accordance with the type of device and current network conditions. QoS parameters like delay time, jitter, bandwidth, trustiness then on are planning to be hold on in an exceedingly\} terribly info which will be used for coaching job intelligent algorithms in a very mobile terminal, thus the $5 \mathrm{G}$ technology can want the foremost effective connections for the device at the given time and condition

\section{B. Psychological feature Radio}

For mobile and wireless communications technologies, since the $4 \mathrm{G}$, ability was a significant issue, as a result of it's to boot applied for $5 \mathrm{G}$. Ability system implies that any system with altogether completely different technologies can work on and communicate with each other. The specification for $5 \mathrm{G}$ mobile system consists of a user terminal and style of freelance, autonomous radio access technologies [6]. Within each of the terminals, each of the radio access technologies is seen as a result of the data science link to the skin internet world [6]. With the employment of psychological feature radio terminal, 5G will do ability and still have a good quality of service. Inside the psychological feature system, the system acknowledges the location, position and condition to check the foremost effective risk for network. With this system, users can want an appropriate network for communication and altogether completely different wireless networks are planning to be able to integrate and communicate with each other via psychological feature radio devices.

psychological feature radio is Associate in Nursing intelligent communication system that l's responsive to its encompassing setting (i.e., outside world), and uses the methodology of understanding-by-building to be told from the setting and adapt its internal states to maths variations inside the incoming RF stimuli by making corresponding changes in sure operative parameters (e.g., transmit-power, carrier-frequency, and modulation strategy) in amount, with two primary objectives in mind: very reliable communication whenever and wherever needed; economical utilization of the spectrum [7].

By that definition, the psychological feature terminal is also a wise terminal with intelligence to choose the correct network from all the current wireless networks [5]. The choice depends on some data like time, demand, and resource. The 5G technology proposes a universal terminal that need to embrace all of the radio predecessor's choices into one device [5]. This terminal convergence is powerfully sustained by the users' wishes and demands; therefore, psychological feature radio becomes the proper 5G terminal candidate [5]. 


\section{TECHNOLOGICAL CHALLENGES}

By understanding the key technologies of 5G, we've got a bent to familiar the technological challenges that within the main lie on the problems concerning security what is more as restricted frequency resources.

\section{A. Security}

Having the ability to scan the accessible spectrum, make a choice from an oversized vary of operational frequencies, modify modulation waveforms, and perform accommodative resource allocation- all of these in real-time- these new psychological feature Radio technology are planning to be able to adapt to an oversized type of radio interference conditions and adaptively select the foremost economical communication mechanisms [8]. However, to boot to the advantages and potential of the psychological feature radio technology, there unit style of challenges related to security, significantly in psychological feature radio terminals. The paradigm of psychological feature radio systems poses a replacement threat on security, like selfish misbehaviors, harmful interference, commissioned user emulation, competition between commissioned users and eavesdropping [9].

There's an opportunity and need to develop a system that able to forestall the misuse inside the very open and granular 237 management that's provided to the radio interface. One amongst them was projected by [8] with a framework referred to as TRIESTE that's transient term for trustworthy Radio Infrastructure for implementing Spectrum Etiquettes. TRIESTE are planning to be able to confirm that radio devices unit exclusively able to access and use the spectrum throughout a way that conforms to their privileges [8].

\section{B. Restricted Frequency Spectrum Resources}

Restricted spectrum resources yielded a major challenge for mobile and wireless technologies. Those restricted frequency and time unit divided to be used among multiple users. Attributable to this condition, itl's expected to spice up efficiency therefore on reinforce the aptitude and quality of the system. To realize this, several multiple access techniques used recently, for example, Time Division Multiple Access (TDMA), Frequency Division Multiple Access (FDMA), Code Division Multiple Access (CDMA), Orthogonal Frequency Division Multiple Access (OFDMA), etc. However, altogether of multiple access system that unit used recently, the aptitude of a mobile communication system depends on time and frequency. This generates a challenge to develop a multiple access system that's able to resolve the dependencies of capability to the restricted frequency spectrum. Korean analysis and development has urged BDMA as a radio interface for 5G, that isn I't trustworthy frequency/time resources [1].

The BDMA technique of this invention divides associate antenna beam in step with locations of the mobile stations to allow the mobile stations to supply multiple accesses, thereby significantly increasing the aptitude of the system [1]. In such a concept, mobile stations and a base station unit throughout a Line of Sight (LOS) state, so as that they exactly perceive each otherl's positions. Throughout this condition theyl'll be able to transmit beams that direct to each otherl's position to talk whereas not interfering with mobile stations at cell edge [1].

For adapting the BDMA system into 5G, the event of the half array antenna is required. The smart antenna with the flexibleness to switch its beam is needed. Switched beam antennas support radio positioning via Angle of Arrival (AOA) data collected from base and mobile stations. The utilization of accommodative antenna arrays is one area that shows probability for rising the capabilities. 


\section{INNOVATION OPPORTUNITIES}

Opening up innovations probability suggests that to seek out the technical area as a result of the steering to conduct analysis work on such specific technological agenda. We've got a bent to monitored the unfold of technical issues related to $5 \mathrm{G}$ in varied scientific and engineering journals. There unit of measurement eighteen analysis articles that have specific $5 \mathrm{G}$ as a result of the most corresponding topic in their analysis work. Those publications embody seven conference papers, seven journal papers, 3 conference reviews and one document review.

Later on, we've got a bent to map the technical area supported forty keywords mentioned in those eighteen analysis articles. Table one lists the keywords and additionally the corresponding style of analysis articles. In general, it within the main indicates that technical area isnl't nonetheless heavily targeting certain technological agenda. However, further researches were conducted concerning topics of relay selection, mobile impromptu network and one hop cooperative mackintosh, whereas completely different topics unit of measurement equally distributed.

We've got a bent to familiar that the keywords unit of measurement likely relevant to a number of technological agenda, i.e. security, network, and technological implementation and applications issues.

\begin{tabular}{|l|l|}
\hline \multicolumn{1}{|c|}{ Keyword on the research articles } & $\begin{array}{c}\text { Number of research } \\
\text { publications }\end{array}$ \\
\hline Relay selection & 4 \\
\hline Mobile Adhoc Networks (MANETS) & 3 \\
\hline One-hop cooperative MAC & 3 \\
\hline Ad hoc On-Demand Distance Vector & 2 \\
\hline Architecture for humanity & 2 \\
\hline Blacklisting & 2 \\
\hline Dnynamic Source Routing (DSR) & 2 \\
\hline Error Vector Magnitude (EVM) & 2 \\
\hline GNU Radio & 2 \\
\hline Multihop wireless network & 2 \\
\hline Orthogonal space time codes & 2 \\
\hline Prognoses & 2 \\
\hline Remote monitoring & 2 \\
\hline Two-hop cooperative communication & 2 \\
\hline Universal Software Radio Peripheral & 2 \\
\hline Wireless optics & 2 \\
\hline All IP networks & 1 \\
\hline Collision avoidance & 1 \\
\hline Constellation sizes & 1 \\
\hline Cooperative protocols & 1 \\
\hline Core networks & 1 \\
\hline Denial of Service (Dos) & 1 \\
\hline Dense network & 1 \\
\hline Heterogeneous networking & 1 \\
\hline Linear complexity & 1 \\
\hline MiMO channels & \\
\hline
\end{tabular}




\begin{tabular}{|l|l|}
\hline Modulation technique & 1 \\
\hline Multi-hops & 1 \\
\hline Network achitecture & 1 \\
\hline OFDM systems & 1 \\
\hline Packet delivery ratio & 1 \\
\hline Parallel transmission & 1 \\
\hline Piraeus & 1 \\
\hline Service oriented architecture & 1 \\
\hline Space diversity & 1 \\
\hline Space time code & 1 \\
\hline Spatial diversity & 1 \\
\hline Spectrum efficiency & 1 \\
\hline Throughput improvement & 1 \\
\hline Ubiquitous and pervasive computing & 1 \\
\hline
\end{tabular}

Table I. Keyword on 5G analysis Publications

Security technological agenda spreads over collision dodging, Denial of Service (DoS), blacklisting, etc. Meanwhile, network technological agenda consists of protocol, all science network, impromptu network, etc. another technological agenda that keep being the foremost researched topic unit of measurement frequency hopping topic, MIMO system, space time codes, relay selection techniques, multi hop systems, etc. There are variety of topics completely different those main topics, that unit of measurement covering regarding implementation of the planned 5G applications, like planted medical devices, remote observation and telemedicine.

Supported those findings, we've got a bent to may formulate the construct to use happen upon certain technological agenda. Researchers might continue those specific areas, which could cause new innovation on 5G. Such an idea is in the main profit the developing country that intends to increase their innovation and technological fight. For example, since relay selection has been typically researched, a replacement man of science may establish the cooperative work with completely different researchers engaged on that field. As inside the long run, wireless network is forecasted will able to support the relay-based communication, where the relay node is well-placed to receive messages from the availability node, process it, then forward it to the node of its meant destination [10]. As another, developing country can place further concentration on another topic like spatial diversity, AODV, or technique to spice up collision dodging, since the works on those fields unit of measurement relatively less conducted.

Because the continuous upgrading of wireless technologies, the basic construct of $5 \mathrm{G}$ is to open up all the doors of potential ways in which, technologies and techniques used to leverage telecommunication for human life. Combining with the angle of 5G key technologies, specifically regarding flat-based science and psychological feature radio, any researchers may contribute to the event of relevant traditional.

Finally, itl's usually famous that technological development of preceding standards $(1 \mathrm{G}$, $2 \mathrm{G}$ and $3 \mathrm{G}$ ) were dominantly controlled by developed countries like USA, Japan and a number of European countries. Therefore, in current and future time, developing countries need to contribute to $5 \mathrm{G}$ technological development by utilizing innovation opportunities. Results of study works on $5 \mathrm{G}$ is also created into technical submission to the international standardization bodies, like ITU. Meanwhile, developing countries might push their national industries to develop patents and to create innovation on relevant $5 \mathrm{G}$ technical areas. it l's 
believed that those schemes may influence the worldwide standards development and increase the country's fight.

\section{CONCLUSION}

Key technologies inside the seam of $5 \mathrm{G}$ has been reviewed, i.e. Flat science primarily based Network and psychological feature Radio. The key technologies facilitate America to spot the 5G technological challenges. The challenges primarily exist inside the protection area and thus the restricted frequency resources downside.

We've got familiar innovation probability about the technological development of 5G standards from understanding technological challenges and exploring basic literature framework. There is a probability and demand to develop a system which will be able to stop the misuse of management, maintain the protection and enhance the aptitude of system. as a result of the results of exploring literature framework, we've got a bent to conclude three technological agenda inside that one may contribute to, i.e. security, network, and technological implementation and applications issues. Consequently, analysers can produce new analysis that specialise in those technical area and build new innovation for the 5G technological development. It is expected that analysis works may result a relevant submission to the international standardization bodies. On the other hand, innovation is together created by pushing national industries to develop patents and to make innovation on such relevant $5 \mathrm{G}$ technical areas.

\section{REFERENCES}

[1] Patil, and P Bhat,।"A Review On 5G Technology \" International Journal of Engineering and Innovative Technology, vol one Issue one, Jan 2012.

[2] A.Tudzarov, and T.Janevski, "Design for 5G Mobile Network Architecture" International Journal of Communication Networks and information Security, vol 3 No 2, August 2011.

[3] C.I. Badoi, N. Prasad, V. Croitiru, and R. Prasad, "5G supported psychological feature Radio" Wireless Pers Communication, 2011.

[4] A.K. Pachauri, and O. Singh, "5G Technology - Redefining Wireless Communication in Upcooming Years" International Journal of field and Management analysis, vol one Issue one, August. 2012.

[5] T. Janevski, "5G mobile Concept" IEEE sixth shopper Communications and Network Conference. 2009.

[6] A. Tudzarov, and T. Janevski, "Functional vogue for 5G Mobile Networks" International Journal of Advanced Science and Technology, vol 32, August 2011.

[7] K.C. Chen and R. Prasad, psychological feature radio networks. the big apple Wiley. 2009.

[8] W. Xu, P. Kamat, W. Trappe, TRIESTE: A trustworthy Radio Infrastructure for implementing Spectrum Etiquettes. Networking Technologies for package made public Radio Networks, 2006. SDR l'06. 1st IEEE Workshop pp.101-109, 25-25 Gregorian calendar month. 2006.

[9] G.A. Safdar and M. O’Neill, “Common management Channel security Framework for psychological feature Radio Networks" IEEE 69th transport Technology Conference. 2009.

[10] C.K. Lo, R.W.Heath, and S. Vishwanath, "The Impact of Channel Feedback on expedient Relay choice for Hybrid-ARQ in Wireless Networks" transport Technology, IEEE. 